Ann. Abeille, I961, 4 (4), 369-376.

\title{
MÉTHODES D'APPRÉCIATION DES SURFACES DE COUVAIN DANS LES COLONIES D'ABEILLES
}

\author{
J. FRESNAYE \\ Station expérimentale d'Apiculture, \\ Centre de Recherches agronomiques du Sud-Est, Montfavet (Vaucluse). \\ Y. LENSKY \\ Université hébrä̈que de Jérusalem, Faculté d'Agriculture, Rehovot (Israël).
}

\section{SOMMAIRE}

Description et comparaison de quatre méthodes pour le calcul de la surface du couvain dans les colonies d'Abeilles en vue de connaître leurs possibilités d'utilisation dans des expériences faisant intervenir un grand nombre de ruches. Les auteurs ont été conduits à éliminer comme non satisfaisantes :

Les méthodes trop lentes, qui perturbent les colonies et ne peuvent pratiquement pas être appliquées à un grand nombre de ruches.

Les méthodes imprécises qui fournissent des résultats erronés et de ce fait inutilisables.

La méthode consistant à mesurer les axes de l'éllipse de couvain qui a été finalement retenue permet le calcul de la surface du couvain en un temps suffisamment court et apporte une précision satisfaisante.

\section{I. - INTRODUCTION}

La surface occupée par le couvain à l'intérieur des ruches conditionne le développement des colonies. En apiculture expérimentale il est indispensable de connaitre cette surface, parfois à plusieurs stades des expériences.

Plusieurs méthodes permettant la mensuration des surfaces de couvain dans les colonies d'abeilles ont été jusqu'ici utilisées et décrites par différents auteurs. La plupart ne peuvent être mises en application que sur un très petit nombre de ruches, en raison de leur lenteur opératoire et de la perturbation qu'elles apportent à la colonie. D'autre part une précision suffisante dans le calcul de la surface du couvain est indispensable à la réalisation d'expériences significatives. C'est pourquoi, dans le but d'évaluer leur possibilité d'emploi et leur précision, nous avons comparé entre elles les méthodes actuellement connues et qui semblent applicables à des expériences faisant intervenir un grand nombre de ruches. 


\section{II. - MÉTHODES D'APPRÉCIATION DU DÉVELOPPEMENT DES COLONIES D'ABEILLES.}

L'étude du développement des populations d'abeilles peut être réalisée à l'aide de deux types de méthodes.

\section{a) Le dénombrement de la population adulte.}

Cette méthode est peu utilisée.

JEFFREE (I957) procède à “ l'estimation " sur photographie du nombre d'abeilles adultes se trouvant sur les cadres d'une ruche. Cette méthode est peu précise et ne permet pas d'apprécier le nombre d'abeilles en vol qui est très variable selon les conditions météorologiques, les ressources mellifères et le moment de la journée.

La pesée des abeilles est plus précise mais difficilement praticable. Il faut anesthésier la colonie et la secouer dans un récipient convenable. La perturbation est énorme et fausse la suite des expériences.

\section{b) L'appréciation des surfaces de couvain}

Ces méthodes sont plus facilement applicables. En outre, elles permettent d'observer l'action de traitements expérimentaux une ou deux semaines plus tôt que les méthodes précédentes. Nous distinguerons divers procédés :

\section{Dénombrement des cadres de couvain.}

Généralement utilisé par l'apiculteur, il est très rapide. Le pourcentage d'erreur qu'il apporte atteint et dépasse $200 \mathrm{p}$. roo, des fluctuations de couvain importantes pouvant se produire sans que varie le nombre des cadres porteurs de couvain (SimpSoN I958). L'expérimentateur ne peut donc l'utiliser en raison de son imprécision.

Estimation visuelle des surfaces de couvain.

Ce procédé est d'une grande rapidité, mais ne représente qu'une amélioration insuffisante de la précédente. L'expérimentateur s'entraîne préalablement à reconnaî-

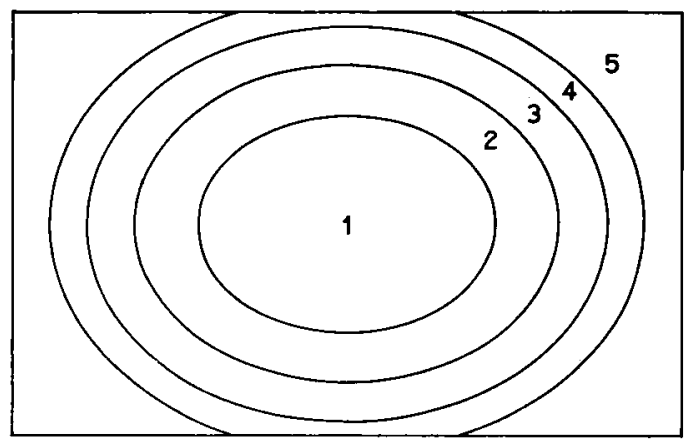

FIG. I, - Ellipses témoins pour la méthode de l'estimation visuelle et comptage par points (réduites).

tre des ellipses de grandeurs différentes dessinées sur des cartons de la taille d'un cadre (fig. I). Cet apprentissage lui permet ensuite d'attribuer aux plaques de couvain une note de I à 5 qu'il additionne avec celles des autres cadres de la ruche. Chaque point a 
une valeur de $200 \mathrm{~cm}^{2}$. La surface totale d'une face de cadre vaut 5 points soit I 000 $\mathrm{cm}^{2}$ environ pour un cadre de ruche "DADANT " normalement construit. L'addition se fait mentalement ce qui évite les calculs ultérieurs.

Nous avons utilisé cette méthode à la Station Expérimentale d'Apiculture de MontFavet sur un grand nombre de ruches a fin de connaître la précocité des colonies et le développement maximum du couvaitı. Nous l'avons abandonnée en raison de sa précision insuffisante pour l'expérimentation.

\section{Calcul de la surface de l'ellipse.}

Les plaques de couvain qui composent le nid à couvain affectent généralement une forme ellipsoïdale ce qui permet d'appliquer la formule très simple du calcul de la surface de l'ellipse (BRUNnich I922). Pour cela on mesure le grand axe et le petit axe à l'aide d'une règle graduée ; il n'est pas nécessaire de seconer les Abeilles se trouvant sur le cadre : généralement leur nombre est insuffisant pour cacher les cellules. La forme de l'ellipse n'est cependant pas toujours respectée par la reine qui pond en fonction des cellules libres et de la forme du cadre; il arrive que des groupes de cellules situées à 1'intérieur de l'ellipse soient vides ce qui détermine un certain pourcentage d'erreur.

Puchta (I949) propose soit une modification de la formule habituelle $\mathrm{S}=4 \times \frac{\mathrm{A}}{2} \times \frac{\mathrm{a}}{2}$ au lieu de $\mathrm{S}=\pi \times \frac{\mathrm{A}}{2} \times \frac{\mathrm{a}}{2}$ soit l'utilisation de tables numériques tenant compte des irrégularités et des cléformations de la surface du couvain. Le pourcentage d'erreur se trouve diminué.

Méthode de Jeffree (1951).

JEFFREE mesure la surface du couvain à l'aide d'un cadre métallique porteur d'ellipses et de lignes diverses qui permettent des corrections. Ce cadre métallique est posé sur le couvain après secouage des Abeilles : on détermine la surface du couvain en choisissant la ligne dont le tracé se rapproche le plus des contours du couvain. On pent soustraire de la surface ainsi définie une certaine fraction si une parcelle intérieure ne comporte pas de couvain. La perturbation causée à la colonie est assez importante. Cette méthode est difficilement applicable à un grand nombre de colonies.

\section{Méthode au cadre divisé en cases par un quadrillage.}

Les cadres de couvain sont introduits verticalement dans une boite spéciale dont les deux faces sont divisées en cases égales par des fils. Un axe permet de faire pivoter la boîte et de présenter alternativement l'une ou l'autre de ses faces. On se sert de fetuilles de papier quadrillé portant la reproduction à l'échelle I /5 du cadre divisé par les fils. L'expérimentateur dessine sur le papier les contours du couvain en prenant les côtés et les angles des différentes cases pour points de repère (fig. 2). La boîte est construite de telle façon qu'il n'est pas nécessaire de secouer les Abeilles des cadres avant de les introduire; les colonies sont ainsi moins perturbées et l'agressivité beaucoup moins élevée. Nous avons utilisé cette méthode à la Station de Montfavet (LouvEauXFRESNAYE, I958, non publié). car elle permet de discriminer les différents stades du 
couvain (cufs, larves, couvain operculé) mais aussi de mesurer la surface et la position du pollen et du miel se trouvant sur les cadres. Il est à noter que dans le cas du pollen et du miel le pourcentage d'erreur est plus élevé car les cellules de pollen sont souvent imparfaitement remplies et les cellules de miel sont de profondeur très variable.

On calcule les surfaces au planimètre ce qui représente un travail assez important qui s'ajoute à celui effectué sur le terrain pour le dessin des surfaces.
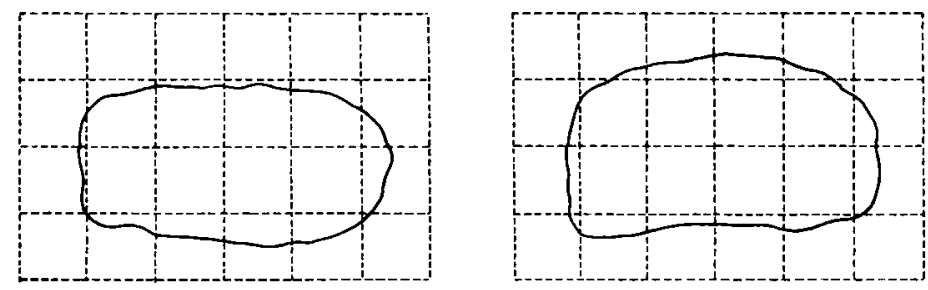

Fig. 2. - Dessins des contours du couvain dans la móthode du cadre dirisé en cases par un quadrillage.

\section{Méthode du dessin des contours du couvain.}

On applique sur le couvain, après secouage de toutes les Abeilles se trouvant sur le cadre, un rectangle de verre ou de plexiglas sur lequel on fixe une feuille de cellophane. On dessine sur cette feuille les contours du couvain de façon très précise avec un crayon gras. On dessine également les parties sans couvain se trouvant à l'intérieur de la surface déjà délimitée afin de pouvoir les soustraire de celle-ci. On change de feuille de cellophane pour chaque face de cadre. Un seul rectangle de verre suffit pour tous les cadres : il sert seulement de support à la cellophane. L,e calcul de la surface se fait au planimètre.

Cette méthode permet de mesurer les surfaces occupées par le couvain aux différents stades (L ENSKY, thèse en cours d'impression) ainsi que les surfaces occupées par le pollen et le miel. Elle est d'une très grande précision mais ne peut être employée sur un grand nombre de ruches en raison de son extrème lenteur et de la perturbation causée à la ruche par la durée de la visite et le secouage de toutes les Abeilles.

\section{Photographie des surfaces de couvain.}

Cette technique nécessite souvent un éclairage artificiel. Après agrandissement des clichés, ou par projection du film, il est possible de compter les cellules habitées (NolaN 1925). Cette méthode est extrèmement lente et impraticable par un observateur isolé sur plus d'une ou deux ruches. ARmbruster (I924) utilisait également les vues photographiques mais il estimait les surfaces à travers un quadrillage ce qui était plus rapide mais aussi moins précis.

\section{Méthode de Chauvin (1950).}

Chauvin a mis au point une méthode permettant de connaître avec précision la ponte journalière d'une reine d'Abeilles sans perturbation pour la colonie. Un rayon, dont une seule face est conservée est collé sur une vitre qui remplace donc le fond des cellules. La reine dépose les œufs sur ce fond, ce qui permet de les compter par transparence de même que les larves et les nymphes. 
Le dénombrement des œufs et des larves ainsi que du pollen est facilité en tendant derrière la ruche des fils qui forment un quadrillage dont les cases sont numérotées. Le relevé case par case est aisé. Cette méthode est une méthode de laboratoire qui n'est utilisable que pour des problèmes spéciaux, intéressant de petites colonies d'Abeilles.

Ce premier examen des méthodes de mesure des surfaces de couvain des colonies d'Abeilles montre que si le problème a été étudié par de nombreux auteurs, les solutions proposées n'en restent pas moins de valeur très inégale. Notre but étant de faire des mesures rapides et précises sur un grand nombre de colonies nous pourrons d'emblée éliminer certaines techniques compliquées ou imprécises. Nous verrons maintenant ce qu'il faut penser de celles que nous avons retenues.

\section{III. - COMPARAISON DE QUEIQQUES MÉTHODES}

Les trois méthodes que nous avons retenues pour le; comparer sont les suivantes:

-- l'estimation visueile;

- le calcul de la surface de l'ellipse;

- la mśthode du cadre divisé en cases par un quadrillage.

Afin de connaître les pourcentages d'erreur inhérents à ces différentes méthodes ous avons pris comme référence la méthode très précise du dessin des contours dun couvain, considérée à priori comme la plus exacte avec la méthode photographique. Les comparaisons ont porté sur la précision, la rapidité et le caractère perturbateur de l'opération.

Nous avons utilisé trois colonies d'Abeilles qui nous ont fourni vingt cadres de couvain auxquels nous avons appliqué successivement les quatre méthodes en chronométrant le temps nécessaire à chaque opération.

I.e tableau I présente les résultats obtenus sur les vingt surfaces expérimentées, la différence en $\mathrm{cm}^{2}$ avec la méthode considérée comme exacte et les pourcentages d'erreur.

Le tableau 2 donne les temps nécessaires à l'application de chaque méthode (temps pour les mesures + temps pour les calculs) par face de cadre. Il donne en outre le temps nécessaire aux calculs de la surface totale du couvain des ruches « Dadant ", de développement moyen, comportant sept cadres de couvain. Il faut ajouter à ce total les temps nécessaires pour l'enfumage, l'ouverture de la ruche, le déplacement des cadres, et éventuellement le brossage des Abeilles.

Avant d'analyser les résultats présentés dans les tableaux nous préciserons quelques points importants :

Les pourcentages d'erreur présentés au bas des colonnes 5-8 et II sont soit en plus soit en moins de la surface réelle du couvain.

Les moyennes arithmétiques ne fournissent qu'une partie des renseignements, et ne tiennent aucun compte de l'amplitude des erreurs qui est parfois considérable. 
Les tableaux I et 2 nous permettent de constater que :

La méthode de l'estimation visuelle est la plus rapide et la plus facile à pratiquer lorsque l'expérimentateur à un certain entraînement. L,e pourcentage d'erreur moyen de $\pm 23,3$ p. Ioo. Pour l'une des mesures il atteint 78,5 p. Ioo et on trouve

TABLEAU I

Résultats des différentes méthodes de mesure de surface du couvain

\begin{tabular}{|c|c|c|c|c|c|c|c|c|c|c|}
\hline 1 & 2 & 3 & 4 & 5 & 6 & 7 & 8 & 9 & 10 & 11 \\
\hline 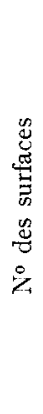 & 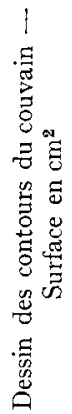 & 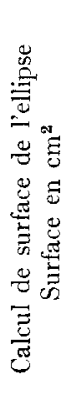 & 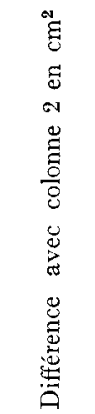 & 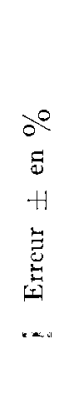 & 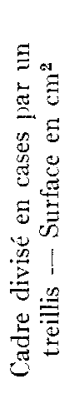 & 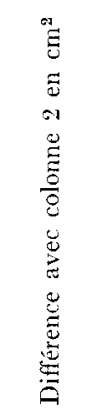 & 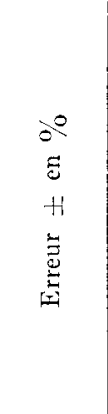 & 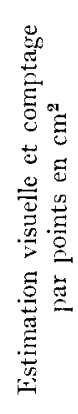 & 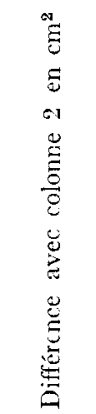 & 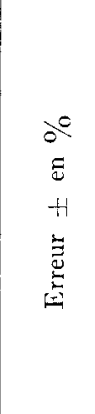 \\
\hline 1 & 584 & 502 & -82 & 14 & 431 & -153 & 26,1 & 600 & $+\quad 16$ & 2,7 \\
\hline 2 & 336 & 386 & +50 & 14,8 & 410 & +74 & 22,0 & 600 & +264 & 78,5 \\
\hline 3 & 634 & 565 & -69 & 10,8 & 535 & -99 & 15,6 & 600 & - 34 & 5,3 \\
\hline 4 & 648 & 549 & $-\quad 99$ & 15,2 & 540 & -108 & 16,6 & 600 & +48 & 7,4 \\
\hline 5 & 385 & 361 & -24 & 6,2 & 455 & +70 & 18,1 & 600 & +215 & 55,8 \\
\hline 6 & 442 & 326 & -116 & 26,2 & 455 & +13 & 2,9 & 600 & +158 & 35,7 \\
\hline 7 & 280 & 285 & +5 & 1,7 & 338 & $\div 58$ & 20,7 & 400 & +120 & 43,0 \\
\hline 8 & 317 & 288 & -25 & 7,8 & 351 & $+\quad 3{ }^{\prime}$ & 10,7 & 400 & $\begin{array}{l}+\quad 83 \\
\end{array}$ & 26,1 \\
\hline 9 & 459 & 477 & $+\quad 18$ & 3,9 & 436 & -23 & 5,0 & ${ }_{4}^{4} 00$ & $+\quad 59$ & 12,8 \\
\hline 10 & 545 & 502 & - 43 & 7,8 & 447 & -102 & 18,7 & 600 & +155 & 28,4 \\
\hline 11 & 515 & 518 & +3 & $0, \overline{5}$ & 431 & $-8^{\prime} t$ & 16,3 & 60 & +85 & 16,5 \\
\hline 12 & 510 & 492 & -18 & 3,5 & 460 & -50 & 5,9 & 600 & +90 & 17,8 \\
\hline 13 & 487 & 612 & +125 & 25,6 & 579 & +92 & 18,8 & 600 & +113 & 23,2 \\
\hline 14 & 641 & 527 & -114 & 17,7 & 514 & -127 & 19,8 & 600 & +41 & 6,3 \\
\hline 15 & 308 & 373 & +65 & 21,1 & $38^{\prime}$ & +76 & 25,3 & $\iota_{t} 00$ & +92 & 29,8 \\
\hline 16 & 317 & 301 & -16 & 5,0 & $37 x$ & +57 & 17,9 & 400 & +83 & 26,1 \\
\hline 17 & 508 & 339 & -169 & 33,2 & 499 & -9 & 2,6 & 600 & +92 & 18,1 \\
\hline 18 & 495 & 361 & -134 & 27,0 & 460 & $-\quad 35$ & 7,0 & 600 & +105 & 21,2 \\
\hline 19 & 583 & 505 & -78 & 13,2 & 535 & - 48 & 8,2 & 600 & +17 & 2,9 \\
\hline 20 & 548 & 480 & $\ldots 68$ & $12, \mathbf{k}$ & 556 & +8 & 1,4 & 600 & +52 & $9,{ }^{\prime}, t$ \\
\hline & \multicolumn{4}{|c|}{ Moyennes Arithmétiques $\pm 13,39$} & & & $\pm 14,18$ & & & $\pm 23,3$ \\
\hline
\end{tabular}

plus communément 30 à $50 \mathrm{p}$. Ioo. L'erreur provient généralement d'une surestimation visuelle, qui est un phénomène optique courant. Ce pourcentage moyen élevé et les risques d'erreurs grossières que comporte la méthode nous la feront éliminer dans tous les ;as où une plus grande précision est nécessaire. Elle ne pourra être utilisée que pour des mesures assez grossières.

La méthode du calcul de la surface de l'eilipse est d'une rapidité assez grande pour être applicable à un nombre de ruches élevé surtout si 1'on opère à deux. Les calculs sont faits ultérieurement; ils demandent d'ailleurs beaucoup plus de temps que les 
TABLEAU 2

Chronométrage du temps nécessaire aux mesures et calculs de surfaces pour les quatre méthodes.

\begin{tabular}{|c|c|c|c|c|c|}
\hline \multirow{2}{*}{ Méthode } & \multicolumn{3}{|c|}{ Chronométrage } & \multirow{2}{*}{$\begin{array}{c}\text { Nombre } \\
\text { d'opérateurs } \\
\text { pour les } \\
\text { mesures }\end{array}$} & \multirow{2}{*}{$\begin{array}{l}\text { Temps } \\
\text { pour une } \\
\text { ruche }\end{array}$} \\
\hline & $\begin{array}{c}\text { Mesure } \\
\text { de surface }\end{array}$ & $\begin{array}{c}\text { Calcul } \\
\text { de surface }\end{array}$ & Total & & \\
\hline $\begin{array}{l}\text { Dessin des contours du } \\
\text { couvain } \ldots \ldots \ldots \ldots \ldots\end{array}$ & $1^{\prime} 35^{\prime \prime}$ & $3^{\prime} 53^{\prime \prime}$ & $5^{\prime} 28^{\prime \prime}$ & 2 & $1 \mathrm{~h} 16$ \\
\hline $\begin{array}{c}\text { Cadre divisé en cases par } \\
\text { un treillis ........... }\end{array}$ & $17^{\prime \prime}$ & $1^{\prime} 12^{\prime \prime}$ & $1^{\prime} 29^{\prime \prime}$ & 2 & $20^{\prime}$ \\
\hline $\begin{array}{r}\text { Calcul de la surfare de } \\
\text { l'ellipse } \ldots \ldots \ldots \ldots \ldots\end{array}$ & $8^{\prime \prime} 6$ & $30^{\prime \prime}$ & $38^{\prime \prime} 6$ & 2 & $9^{\prime}$ \\
\hline $\begin{array}{l}\text { Estimation visuelle et } \\
\text { comptage par points.. }\end{array}$ & $3^{\prime \prime}$ & 0 & $3^{\prime \prime}$ & 1 & $1^{\prime}$ \\
\hline
\end{tabular}

mesures. La moyenne des erreurs \pm I3,3 p. Ioo et un maximum rarement approché de 33,2 p. Ioo ne sont pas incompatibles avec l'expérimentation en biologie. Ces erreurs ont surtout pour origine l'irrégularité des ellipses et de la ponte de la reine. L'utilisation de très bonnes colonies diminue ces irrégularités.

La méthode du cadre divisé en cases par un quadrillage est d'une précision voisine de celle de la méthode du calcul de la surface de l'ellipse $( \pm$ I4,8 r p. Ioo d'erreur moyenne), mais elle est plus lente à pratiquer. Le dessin des surfaces sur un quadrillage à grandeur réelle réduirait le pourcentage d'erreur mais serait probablement légèrement plus long à mettre en œuvre. Cette méthode présente l'avantage complémentaire de permettre le caicul des surfaces de miel et de pollen stockés ou leur disposition sur les cadres.

Le temps important nécessité par la méthode du dessin des contours du couvain qui nous a servi de référence montre bien qu'on ne peut l'employer pour des expériences réunissant de nombreuses ruches. D'autre part la perturbation causée aux colonies par le brossage de toutes les Abeilles et par la durée de la visite peut influer beaucoup sur le comportement et les conditions physiologiques ultérieures des colonies. Le brossage des abeilles peut être évité dans les trois méthodes expérimentées : les différences de perturbation se réduisent alors à la durée de la visite qui est variable d'une méthode à 1'a11tre.

\section{IV. - CONCLUSION}

La précision des méthodes de calcul des surfaces de couvain dans les ruches est généralement en rapport avec le temps nécessaire à leur mise en cuvre. Les méthodes précises, (moins de \pm Io p. Ioo d'erreur moyenne) ne sont pas applicables à l'expérimentation sur de nombreuses ruches en raison de leur lenteur ; les méthodes imprécises plus de $\pm 20 \mathrm{p}$. Ioo ) en raison de cette imprécision ne le sont pas non plus. Le calcul des surfaces des ellipses de couvain se place à l'intérieur de ces limites et est actuellement la méthode la plus facilement applicable. 
En partant de ces bases nous envisageons de rechercher des méthodes nouvelles permettant d'augmenter la rapidité des mesures et de réduire le pourcentage d'erreur.

Rę̧u pour publication en avril I961.

\section{SUMMARY}

Description and comparison of four methods of calculating the surface of brood in beecolonies in order to know how these methods can be used in experiments dealing with a large number of hives. The authors had to reject :

I) Methods which are too slow, disturb colonies and can't be practically applied to a large number of hives,

2) Unprecise methods giving erroneous results and thus unuseful. The method chosen is the measuring of the two axis of the brood ellipsis. This method allows us to measure the brood surface rather quikly and give us satisfactory precision.

\section{RÉFÉRENCES BIBLIOGRAPHIQUES}

ARMBruster L., I924-I925. Versuche und Zahlen zum Bienen Brutgeschäft. Arch. Bienenk., 6, 44-57.

JRunnich J., I922. Graphische Darstellung Legetäligkeit einer Bienenkönigin. Arch. Bienenk., 4, I 3 r-I4r.

Chatvin R., i950. Méthode d'observation continue de la ponte de la reine chez les Abeilles. Apic. Sect. Scient., nov.

Cirauvin R., I956. Les facteurs qui gouvernent la ponte chez la reine des Abeilles. Ins. Soc., 3, 499-504.

Chauvin R., I960. Progrès récents dans la biologie de l'Abeille. Ann. Abeille, 1, 5-35.

JEFFREE L. P., I95I. A photographic presentation of estimated numbers of honeybees (Apis miellifera L.) on combs in $14 \times 8 \mathrm{r} / 2$ inch frames. Bee World, 32, $89-9 \mathrm{I}$.

JEFFREE E. P., I957. A shaped wire grid for estimation of the quantities of brood and pollen in comb. Bee World, 39, 32-34.

LENSKY Y. (Thèse non publiée).

Tolan W. J., I925. The brood rearing cycle of the honeybee. U. S. D. A. Dept. Bull, no I 349 .

Ptchta R., I949. Brutnestmessung einfach und schnell. Arch. Bienenk., 26, 30-3I.

Simpson J., 1958. The factors which cause colonies of Apis mellifica to swarm. Ins. Soc., 5, 77-93. 